\title{
Efeito da Heparina Liofilizada e da Líquida sobre a Medida do pH do Sangue do Cordão Umbilical
}

Effect of Lyophilized and Liquid Heparin on Umbilical Cord Blood pH Measure

José Mauro Madi, Edson Nunes de Morais, José Roberto Festugatto, Carlos Leonardo Treméa, Márcio Rigotto

\section{RESUMO}

Objetivo: avaliar o efeito da heparina liofilizada e da liquida sobre a medida do $\mathrm{pH}$ do sangue do cordão umbilical.

Métodos: estudo prospectivo que incluiu cento e duas amostras de sangue arterial do cordão umbilical de recém-nascidos no Serviço de Obstetricia do Hospital Geral de Caxias do Sul. As amostras foram divididas em 51 seringas previamente preparadas com heparina liofilizada (seringa A) e 51 seringas preparadas com heparina liquida (seringa B) no momento da coleta sangüinea. A obtenção das amostras foi realizada por duplo clampeamento do cordão entre pinças, imediatamente após o desprendimento fetal. $\mathrm{O}$ pH arterial do sangue obtido foi analisado em no máximo 20 minutos, em analisador de pH da marca AVL OMNI(Viena, Áustria).

Resultados: a média dos valores de $p H$ no sangue contido nas seringas dos grupos $A$ e $B$ foi de

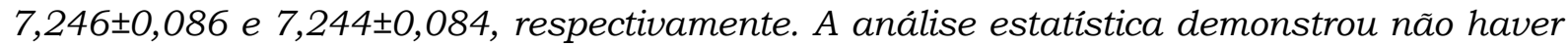
diferença significante entre os valores de $\mathrm{pH}$ entre as amostras de sangue contidas em ambos os conjuntos de seringas.

Conclusão: as duas formas de heparinização de seringas assemelham-se quanto aos efeitos sobre o $\mathrm{pH}$ do sangue dos vasos umbilicais, o que permite a escolha daquela de menor custo.

PALAVRAS-CHAVE: Gasometria. Equilíbrio ácido básico. Heparina.

\section{Introdução}

Desde a década passada, a análise de $\mathrm{pH}$ e gases do sangue dos vasos umbilicais, quando do nascimento, tem sido valorizada para medida da oxigenação e do equilíbrio ácido-básico fetal. Entretanto, o tempo despendido entre a coleta e a realização do exame, a estocagem e o tipo de heparinização das seringas usadas para este fim têm sido motivo de análise e discussão.

O estudo gasométrico do sangue do cordão umbilical deve ser realizado imediatamente após o parto1, e a demora no clampeamento do segmento funicular pode resultar em significantes

Serviço de Obstetrícia do Hospital Geral de Caxias do Sul Fundação Universidade de Caxias do Sul - RS.

Correspondência:

José Mauro Madi

Fundação Universidade de Caxias do Sul - Hospital Geral de Caxias do Sul

Av. Prof. Antônio Vignolli, 255 - Bairro Petrópolis

95001-970 - Caxias do Sul - RS

e-mail: madi@visao.com.br modificações no $\mathrm{pH}, \mathrm{pCO} 2$ e déficit de base. No entanto, esses fatores podem não ser significativos para alterar os valores de gasometria.

Merece discussão, entretanto, o tipo de seringa a ser usado na coleta do sangue funicular, ainda que aquelas previamente heparinizadas com heparina liofilizada proporcionem, segundo alguns autores, resultados mais uniformes e consistentes, minimizando desta forma o erro1. Em contrapartida, a aspiração da heparina líquida pode acarretar resultados falsos, se muita heparina ou quantidade insuficiente de sangue forem aspirados pela seringa.

Tendo em vista a diferença de custo entre os dois tipos de seringas, com heparina liofilizada ou líquida, e sendo os dados de importância para os Serviços de Obstetrícia que estiverem em vias de implantação do uso rotineiro dessa avaliação gasométrica, comparamos nesse estudo os efeitos do tipo de heparinização sobre o $\mathrm{pH}$ do sangue dos vasos do cordão umbilical, coletado imediatamente após o parto. 
Material e Métodos

Foram coletadas 102 amostras de sangue da artéria do cordão umbilical de recém-nascidos no Serviço de Obstetrícia do Hospital Geral de Caxias do Sul (HG-UCS), durante o ano de 2001. As amostras foram divididas, utilizandose seringas previamente preparadas com heparina liofilizada, da marca Becton Dickinson (seringa A), tipo Preset $1 \mathrm{~mL}(\mathrm{n}=51)$, e da marca Plascalp Unijet (seringa $B)(n=51)$, preparadas com heparina líquida no momento da coleta sangüínea. A obtenção das amostras foi realizada por duplo clampeamento do cordão entre pinças, imediatamente após o desprendimento fetal. Após a secção do segmento do cordão e colocação sobre a mesa auxiliar, puncionaram-se artéria e veia umbilicais com os dois tipos de seringas citadas, obtendo-se $2 \mathrm{~mL}$ de sangue de cada vaso. A finalidade da dupla punção de vasos foi identificar corretamente o sangue da artéria umbilical, pois sabe-se que este possui $\mathrm{pH}$ inferior ao da veia (pelo menos 3 unidades $\mathrm{pH}$ ). O tempo gasto entre uma e outra aspiração foi o necessário para a obtenção da amostra da primeira e da segunda seringa, isto é, imediatamente após. $\mathrm{O} \mathrm{pH}$ arterial do sangue obtido foi analisado pelo laboratório de Análises Clínicas do HGFUCS, em no máximo 20 minutos, em aparelho da marca AVL OMNI (Viena, Áustria).

Os resultados do $\mathrm{pH}$ foram analisados comparando-se os seus valores médios por meio do teste $t$ de Student, considerando-se como significante $\mathrm{p}<0,05$.

Esse estudo não apresentou riscos aos indivíduos envolvidos; mesmo assim, obteve-se aprovação da Comissão de Ética e da Comissão Editorial e Científica do Hospital Geral de Caxias do Sul.

\section{Resultados}

A média dos valores da $\mathrm{pH}$ de artéria umbilical do sangue da seringa A foi de $7,246 \pm$ 0,086 , enquanto que a média dos valores obtidos com o uso da seringa B foi de $7,244 \pm 0,084$. A análise estatística demonstrou não haver diferença significante entre os valores de $\mathrm{pH}$ da artéria umbilical do sangue contido nos dois tipos de seringa.

\section{Discussão}

A literatura é pródiga em análises e resultados discordantes sobre o assunto em discussão. Carter et al.2, preocupados com a possibilidade de eventuais erros diagnósticos provocados pelo método de coleta sangüinea, estudaram 71 pacientes submetidos a tratamento intensivo; para tal, dosaram o $\mathrm{pCO}_{2}, \mathrm{pO}_{2}, \mathrm{pH}$, sódio, potássio e glicose, obtidos com dois tipos de seringas (Johns, Hardie Health Care Products Pty Ltd. e Marksman, Martell Medical Products Inc). Observaram que as análises do $\mathrm{pH}$, sódio, potássio e glicose não mostraram diferenças significantes em função dos dois tipos de seringas. Entretanto, diferenças foram observadas na dosagem da $\mathrm{pCO}_{2}$ e $\mathrm{pO}_{2}$, nas amostras coletadas com estas mesmas seringas, quando a $\mathrm{pO}_{2}$ foi menor que $100 \mathrm{mmHg}$.

Hamilton et al.3 observaram que a adição de heparina produziu erro na determinação de $\mathrm{pO}_{2}, \mathrm{pCO}_{2}$ e $\mathrm{pH}$. A dosagem da $\mathrm{pCO}_{2}$ foi a mais afetada, já que a heparina tende a completar o "espaço morto" da seringa e da agulha.

Gayed et al.4 compararam os efeitos da heparina líquida e vaporizada em 35 amostras de sangue obtidas de recém-natos. Não observaram diferença estatística na avaliação de $\mathrm{pH}$ e $\mathrm{pO}_{2}$, mas a $\mathrm{pCO}_{2}$ foi estatisticamente mais baixa com a heparina liquida. Os autores concluem que, se por erro, mais heparina que o necessário for usada, efeitos importantes poderão ser observados; sugerem, também, o uso de seringas com heparina vaporizada, de forma a se obterem resultados confiáveis e consistentes.

Ordog et al.5 avaliaram as alterações em amostras de sangue arterial de 50 pacientes, causadas pela adição de heparina líquida. Usaram, para tal, seringas diferentes e quantidades diferentes de heparina, e observaram que $0,025 \mathrm{~mL}$ da solução permaneceu aderido à seringa. Esta solução remanescente causaria uma chance de erro de 1,25\% nos resultados, aceitável em se considerando que é geralmente menor que o desvio padrão dos resultados laboratoriais. Concluem que o excesso de heparina líquida pode produzir resultados falsos, tais como acidose metabólica, e recomendam a retirada do excesso do produto.

Crockett et al.6 compararam o uso de seringas com heparina liofilizada ou líquida na análise de ácido-base do sangue arterial. Não observaram diferenças estatisticamente significantes. Os nossos resultados, como os desses autores, também não apresentaram diferenças significantes entre as medidas de $\mathrm{pH}$. 
Do ponto de vista de resultados de gasometria, parece não haver diferença entre a utilização de uma ou de outra seringa. Por outro lado, a diferença de custos entre uma e outra é significativa, posto que o valor da seringa $A$ é de US\$ $0.65(R \$ 2,30)$ e da B é de US\$ $0.05(R \$ 0,20)$. $\mathrm{O}$ valor do dólar em relação ao real, por ocasião da elaboração do texto, era de $\mathrm{R} \$ 3,50$.

Entendemos que algumas considerações gerais mereçam especial atenção. Em primeiro lugar, a análise bioquímica do sangue do cordão umbilical deve ser feita antes da primeira incursão inspiratória do recém-nascido. Em segundo, o pinçamento do cordão deve ocorrer imediatamente após o desprendimento fetal, pois evitaria a primeira respiração do recémnato antes do clampeamento. Finalmente, é referido que tempo de permanência das amostras inferior a 60 minutos, em temperatura ambiente, não altera os valores a serem medidos. Recomendamos que em caso de impossibilidade de efetuarem-se as medidas nesse tempo, o armazenamento das seringas deva ser realizado em recipiente com gelo ou em geladeira até a análise.

Com base no presente estudo recomendamos a utilização de seringas preparadas com heparina líquida no momento da coleta, levando em conta os princípios para a minimização dos fatores de erro, tais como quantidade de heparina aspirada, primeira respiração do nascituro, tempo decorrido entre a coleta e a análise, e armazenamento adequado do material.

\section{ABSTRACT}

Purpose: to evaluate the effect of lyophilized and liquid heparin on umbilical cord blood $\mathrm{pH}$ measure.

Method: we carried out a prospective study with 102 newborn arterial umbilical cord blood samples at the Obstetrics Department of the "Hospital Geral de Caxias do Sul". The authors used 51 syringes previously prepared with lyophilized heparin (syringe A) and 51 syringes prepared with liquid heparin (syringe B) for sample collection. Immediately after delivery a segment of the umbilical cord was doubly clamped and blood samples were obtained for measurements of $\mathrm{pH}$ and gases. The umbilical arterial blood $\mathrm{pH}$ was analyzed within 20 minutes by an AVL OMNI $p H$ analyzer (Vienna, Austria) .

Results: the average values of umbilical cord arterial blood $\mathrm{pH}$ in groups $A$ and $B$ were $7.246 \pm 0.086$ and $7.244 \pm 0.084$, respectively. The analysis showed no significant differences between blood samples in both syringes.

Conclusion: we conclude that the two types of syringes have similar effects on blood $\mathrm{pH}$ analysis, allowing us to choose the less expensive one.

KEYWORDS: Gasometry. Acid-base balance. Heparin.

\section{Referências}

1. Thorp, JA, Dildy GA, Yeomans ER, Meyer BA, Parisi VM. Umbilical cord blood gas analysis at delivery. Am J Obstet Gynecol 1996; 175:517-22.

2. Carter BG, Tibballs J, Hochmann M, Osborne A, Chiriano A, Murray G. A comparison of syringes to collect blood for analysis of gases, electrolytes and glucose. Anaesth Intensive Care 1994; 22:698-702.

3. Hamilton RD, Crockett RJ, Alpers JH. Arterial blood gas analysis: potential errors due to the addition of heparin. Anaesth Intensive Care 1978; 6:251-5.

4. Gayed AM, Marino ME, Dolanski EA. Comparison of the effects of dry and liquid heparin on neonatal arterial blood gases. Am J Perinatol 1992; 9:159-61.

5. Ordog GJ, Wasserberger J, Balasubramaniam S. Effect of heparin on arterial blood gases. Ann Emerg Med 1985; 14:233-8.

6. Crockett AJ, McIntyre E, Ruffin R, Alpers JH. Evaluation of lyophilized heparin syringes for the collection of arterial blood for acid base analysis. Anaesth Intensive Care 1981; 9:40-2. 